\title{
Measurement Feedback Self-Tuning Weighted Measurement Fusion Kalman Filter for Systems with Correlated Noises
}

\author{
Xin Wang and Shu-Li Sun \\ Department of Automation, Heilongjiang University, Harbin 150080, China \\ Correspondence should be addressed to Xin Wang, wangxin@hlju.edu.cn
}

Received 26 February 2012; Accepted 19 March 2012

Academic Editor: Baocang Ding

Copyright (c) 2012 X. Wang and S.-L. Sun. This is an open access article distributed under the Creative Commons Attribution License, which permits unrestricted use, distribution, and reproduction in any medium, provided the original work is properly cited.

For the linear discrete stochastic systems with multiple sensors and unknown noise statistics, an online estimators of the noise variances and cross-covariances are designed by using measurement feedback, full-rank decomposition, and weighted least squares theory. Further, a self-tuning weighted measurement fusion Kalman filter is presented. The Fadeeva formula is used to establish ARMA innovation model with unknown noise statistics. The sampling correlated function of the stationary and reversible ARMA innovation model is used to identify the noise statistics. It is proved that the presented self-tuning weighted measurement fusion Kalman filter converges to the optimal weighted measurement fusion Kalman filter, which means its asymptotic global optimality. The simulation result of radar-tracking system shows the effectiveness of the presented algorithm.

\section{Introduction}

With the development of scientific technology, the scale of a control system has become more and more complex and tremendous, and the accuracy, fault-tolerance, and robustness of a system are required much higher, so that single sensor has been unable to satisfy the demands of high scientific technologies. Thus, the multisensor information fusion technology has been paid great attention to and become an important research issue.

In early 1980s, Shalom [1,2] presented the computation formula of cross-covariance matrix by studying the correlation of two sensor subsystems with independent noises. Carlson [3] presented the famous federated Kalman filter by using the upper bound of noise variance matrix to replace noise variance matrix and supposing that the initial local estimation errors are not correlated. Kim [4] proposed the maximum likelihood fusion estimation algorithm by requiring the hypothesis that random variables obey normal distributions. 
The universal weighted least squares method and the best linear unbiased fusion estimation algorithm were presented by $\mathrm{Li}$ et al. [5] on the basis of a unified linear model for three estimation fusion architectures of centralized filter, distributed filter, and hybrid filter. Three weighted fusion algorithms of matrix-weighted, diagonal-matrix-weighted, and scalarweighted in the linear minimum variance sense were proposed by Sun and Deng [6], Sun [7], where the matrix-weighted fusion algorithm, maximum likelihood fusion algorithm [4], and distributed best linear unbiased estimation algorithm [5] have the same result and avoid the derivation on the basis of hypothesis of normal distribution and linear model. The shortcomings of methods presented in [3-7] are that they have larger calculation burdens and the fusion accuracy is global suboptimal.

Based on Kalman filtering, Gan and Chris [8] discussed two kinds of multisensor measurement fusion method: the centralized measurement fusion (CMF) and the weighted measurement fusion (WMF). The former is to directly merge the multisensor data through the augmented measurement vector to calculate the estimation. Its advantage is that it can obtain globally optimal state estimator. Its shortcoming is that the computational burden is large since the measurement dimension is high. So it is unsuitable for real-time application. The latter is to weigh local sensor measurements to obtain a low-dimensional measurement equation, and then to use a single Kalman filter to obtain the final fused state estimation. Its advantages are that the computational burden can be obviously reduced and the globally optimal state estimation can be obtained [8-12].

It is known that the existing information fusion Kalman filtering is only effective when the model parameters and noise statistics are exactly known. But this restricts its applications in practice. In real applications, the model parameters and noise statistics are completely or partially unknown in general. The filtering problem for systems with unknown model parameters and/or noise statistics yields the self-tuning filtering. Its basic principle is the optimal filter plus a recursive identifier of model parameters and/or noise statistics [13].

For self-tuning fusion filters, there are two methods of self-tuning weighted state fusion and self-tuning measurement fusion. Weighted state fusion method is used by Sun [14] and Deng et al. [15], respectively, but the used distributed state fusion algorithm is globally suboptimal and the acquired self-tuning estimator cannot reach globally asymptotic optimality. For the self-tuning measurement fuser, $[9,16]$ considered the uncorrelated input noise and measurement noise. Ran and Deng [17] presented a self-tuning measurement fusion Kalman filter under the assumption that all sensors have the same measurement matrices.

This paper is concerned with the self-tuning filtering problem for a multisensor system with unknown noise variances, different measurement matrices, and correlated noises. Firstly, transform the system with correlated input noise and measurement noise into one with uncorrelated input noise and measurement noise by using the measurement feedback and taking measurement data as a part of system control item. Then, weigh all the measurements by using full-rank decomposition and weighted least squares theory. The Fadeeva formula is used to establish ARMA innovation model with unknown noise covariance matrices and the sampling correlated function of a stationary and reversible ARMA innovation model is used to identify the noise covariance matrices. It is rigorously proved that the presented self-tuning weighted measurement fusion Kalman filter converges to the optimal weighted measurement fusion Kalman filter, that is, it has asymptotic global optimality. 


\section{Problem Formulation}

Consider the controlled multisensor time-invariant systems with correlated noises:

$$
\begin{gathered}
x(t+1)=\Phi x(t)+B u(t)+\Gamma w(t), \\
y_{i}(t)=H_{i} x(t)+v_{i}(t), \quad i=1, \ldots, L,
\end{gathered}
$$

where $x(t) \in R^{n}$ is the state, $u(t) \in R^{p}$ is the given control, $y_{i}(t) \in R^{m_{i}}$ is the measurement of the sensor $i, w(t) \in R^{r}$ is the input noise, and $v_{i}(t) \in R^{m_{i}}$ is the measurement noise. $L$ is the number of sensors, $H_{i} \in R^{m_{i} \times n}$ is the measurement matrix of the sensor $i$. $\Phi, B$, and $\Gamma$ are constant matrices with compatible dimensions.

Assumption 2.1. $w(t)$ and $v_{i}(t)$ are correlated Gaussian white noise with zero means, and

$$
E\left\{\left[\begin{array}{c}
w(t) \\
v_{i}(t)
\end{array}\right]\left[w^{\mathrm{T}}(k) v_{j}^{\mathrm{T}}(k)\right]\right\}=\left[\begin{array}{cc}
Q_{w} & S_{j} \\
S_{i}^{\mathrm{T}} & R_{i j}
\end{array}\right] \delta_{t k}, \quad i=1, \ldots, L,
$$

where the symbol $E$ denotes the expectation, $\delta_{t k}$ is Kronecker delta function, that is, $\delta_{t t}=1$, $\delta_{t k}=0(t \neq k)$. The variance matrix of $v_{i}(t)$ is $R_{i i}=R_{i}$. Combining $L$ measurement equations of (2.2) yields

$$
y^{(\mathrm{I})}(t)=H^{(\mathrm{I})} x(t)+v^{(\mathrm{I})}(t)
$$

where $y^{(\mathrm{I})}(t)=\left[y_{1}^{\mathrm{T}}(t), \ldots, y_{L}^{\mathrm{T}}(t)\right]^{\mathrm{T}}, H^{(\mathrm{I})}=\left[H_{1}^{\mathrm{T}}, \ldots, H_{L}^{\mathrm{T}}\right]^{\mathrm{T}}$ and $v^{(\mathrm{I})}(t)=\left[v_{1}^{\mathrm{T}}(t), \ldots, v_{L}^{\mathrm{T}}(t)\right]^{\mathrm{T}}$. Let the variance of $v^{(\mathrm{I})}(t)$ be $R^{(\mathrm{I})}=\left(R_{i j}\right)>0$ and the cross covariance of $w(t)$ and $v^{(\mathrm{I})}(t)$ be $S=\left[S_{1}, \ldots, S_{L}\right]$.

Assumption 2.2. $\left(\Phi, H^{(\mathrm{I})}\right)$ is a detectable pair and $(\Phi, \Gamma)$ is a controllable pair, or $\Phi$ are stable. Assumption 2.3. Measurement data $y_{i}(t)$ is bounded, that is,

$$
\left\|y_{i}(t)\right\|<c, \quad i=1, \ldots, L,
$$

where $\|\cdot\|$ is the norm of a vector and $c>0$ is a positive real number.

\subsection{CMF and WMF Kalman Filter}

To convert the systems (2.1) and (2.4) into the uncorrelated system, (2.1) is equivalent to

$$
x(t+1)=\Phi x(t)+B u(t)+\Gamma w(t)+J\left[y^{(\mathrm{I})}(t)-H^{(\mathrm{I})} x(t)-v^{(\mathrm{I})}(t)\right],
$$

where $J$ is a pending matrix. (2.6) can be converted into

$$
x(t+1)=\bar{\Phi} x(t)+\bar{u}(t)+\bar{w}(t),
$$


where $\bar{\Phi}=\Phi-J H^{(\mathrm{I})}, \bar{u}(t)=B u(t)+J y^{(\mathrm{I})}(t), \bar{w}(t)=\Gamma w(t)-J v^{(\mathrm{I})}(t) . J y^{(\mathrm{I})}(t)$ as an output feedback becomes a part of the control item. Then, primary system formulae (2.1) and (2.2) are equivalent to the system formed by formulae (2.4) and (2.7). To make $E\left[\bar{w}(t) v^{(\mathrm{I}) \mathrm{T}}(t)\right]=0$, introduce $J=\Gamma S R^{(\mathrm{I})-1}$ which ensures that $\bar{w}(t)$ and $v^{(\mathrm{I})}(t)$ are not correlated. Then, variance matrix of $\bar{w}(t)$ is yielded as $\bar{Q}_{w}=\Gamma\left(Q_{w}-S R^{(\mathrm{I})} S^{\mathrm{T}}\right) \Gamma^{\mathrm{T}}$. From [18], we know that any nonzero matrix $H^{(\mathrm{I})}$ has full-rank decomposition:

$$
H^{(\mathrm{I})}=F H^{(\mathrm{II})}
$$

where $F$ is a full column-rank matrix with the rank $r$, and $H^{(\mathrm{II})}$ is a full row-rank matrix with the rank $r$, then measurement model (2.4) can be represented as

$$
y^{(\mathrm{I})}(t)=F H^{(\mathrm{II})} x(t)+v^{(\mathrm{I})}(t)
$$

Given that $F$ is a full column-rank matrix, it follows that $F^{\mathrm{T}} R^{(\mathrm{I})} F$ is nonsingular. Then, the weighted least squares (WLS) [19] method is used and the Gauss-Markov estimate of $H^{(\mathrm{II})} x(t)$ is yielded as

$$
y^{(\mathrm{II})}(t)=\left(F^{\mathrm{T}} R^{(\mathrm{I})-1} F\right)^{-1} F^{\mathrm{T}} R^{(\mathrm{I})-1} y^{(\mathrm{I})}(t),
$$

substituting (2.9) into (2.10) yields

$$
\begin{gathered}
y^{(\mathrm{II})}(t)=H^{(\mathrm{II})} x(t)+v^{(\mathrm{II})}(t), \\
v^{(\mathrm{II})}(t)=\left(F^{\mathrm{T}} R^{(\mathrm{I})-1} F\right)^{-1} F^{\mathrm{T}} R^{(\mathrm{I})-1} v^{(\mathrm{I})}(t) .
\end{gathered}
$$

The variance matrix $R^{(\mathrm{II})}=\mathrm{E}\left[v^{(\mathrm{II})}(t) v^{(\mathrm{III)} T}(t)\right]$ of $v^{(\mathrm{II})}(t)$ is given by

$$
R^{(\mathrm{II})}=\left(F^{\mathrm{T}} R^{(\mathrm{I})-1} F\right)^{-1}
$$

For systems (2.4) and (2.7), and (2.7) and (2.11), respectively, using standard Kalman filtering algorithm [20], we can obtain CMF and WMF Kalman estimators $\widehat{x}^{(i)}(t \mid t+j), i=\mathrm{I}$, II, $j=0, j<0$, and $j>0$, and their error variance matrices $P^{(i)}(t \mid t+j)$. It is proved in [11] that the weighted measurement fusion steady-state Kalman filter $\widehat{x}^{(I I)}(t \mid t)$ for the weighted measurement fusion system (2.7) and (2.11) has the global optimality, that is, it is numerically identical to the CMF steady-state Kalman filter $\widehat{x}^{(\mathrm{I})}(t \mid t)$ if they have the same initial values.

The above WMF method can obviously reduce the computational burden since the dimension of the measurement vector for the centralized measurement fusion is $m \times 1, m=$ $m_{1}+m_{2}+\cdots+m_{L}$, while that for the weighted measurement fusion is $r \times 1$, and $m$ is much larger than $r$ generally. 


\subsection{Optimal Measurement Fusion Steady-State Kalman Filter}

By the above WMF methods, the corresponding optimal steady-state Kalman filter is given as [21]

$$
\begin{gathered}
\widehat{x}^{(\mathrm{II})}(t+1 \mid t+1)=\widehat{x}^{(\mathrm{II})}(t+1 \mid t)+K_{f}^{(\mathrm{II})} \varepsilon^{(\mathrm{II})}(t+1), \\
\widehat{x}^{(\mathrm{II})}(t+1 \mid t)=\Psi_{p}^{(\mathrm{II})} \widehat{x}^{(\mathrm{II})}(t \mid t-1)+\bar{K}_{p}^{(\mathrm{II})} y^{(\mathrm{II})}(t)+\bar{u}(t), \\
\widehat{x}^{(\mathrm{II})}(t \mid t+N)=\widehat{x}^{(\mathrm{II})}(t \mid t)+\sum_{j=1}^{N} K^{(\mathrm{II})}(j) \varepsilon^{(\mathrm{II})}(t+j), \quad N>0, \\
\bar{u}(t)=B u(t)+J y^{(\mathrm{II})}(t), \\
\varepsilon^{(\mathrm{II})}(t)=y^{(\mathrm{II})}(t)-H^{(\mathrm{II})} \widehat{x}^{(\mathrm{III})}(t \mid t-1), \\
K_{f}^{(\mathrm{II})}=\Sigma^{(\mathrm{II})} H^{(\mathrm{II}) \mathrm{T}}\left(H^{(\mathrm{II})} \Sigma^{(\mathrm{II})} H^{(\mathrm{II}) \mathrm{T}}+R^{(\mathrm{II})}\right)^{-1}, \\
\bar{K}_{p}^{(\mathrm{II})}=\bar{\Phi} \Sigma^{(\mathrm{II})} H^{(\mathrm{II}) \mathrm{T}}\left(H^{(\mathrm{II})} \Sigma^{(\mathrm{II})} H^{(\mathrm{II}) \mathrm{T}}+R^{(\mathrm{II})}\right)^{-1}, \\
\Psi_{p}^{(\mathrm{II})}=\bar{\Phi}-\bar{K}_{p}^{(\mathrm{II})} H^{(\mathrm{II})}, \\
K^{(\mathrm{II})}(j)=\Sigma^{(\mathrm{II})}\left(\left(I_{n}-K_{f}^{(\mathrm{II})} H^{(\mathrm{II})}\right)^{\mathrm{T}} \bar{\Phi}^{\mathrm{T}}\right)^{j} H^{(\mathrm{II}) \mathrm{T}}\left(H^{(\mathrm{II})} \Sigma^{(\mathrm{II})} H^{(\mathrm{II}) \mathrm{T}}+R^{(\mathrm{II})}\right)^{-1}, \\
P^{(\mathrm{II})}=\left[I_{n}-K_{f}^{(\mathrm{II})} H^{(\mathrm{II})}\right] \Sigma^{(\mathrm{II})},
\end{gathered}
$$

where $\Psi_{p}^{(\mathrm{II})}$ is a stable matrix [19] and $\Sigma^{(\mathrm{II})}$ satisfies the following Riccati equation:

$$
\Sigma^{(\mathrm{II})}=\bar{\Phi}\left[\Sigma^{(\mathrm{II})}-\Sigma^{(\mathrm{II})} H^{(\mathrm{II}) \mathrm{T}}\left(H^{(\mathrm{II})} \Sigma^{(\mathrm{II})} H^{(\mathrm{II}) \mathrm{T}}+R^{(\mathrm{II})}\right)^{-1} H^{(\mathrm{II})} \Sigma^{(\mathrm{II})}\right] \bar{\Phi}^{\mathrm{T}}+\bar{Q}_{w} .
$$

When noise variance matrices $Q_{w}, S_{i}$, and $R_{i j}(i, j=1, \ldots, L)$ are unknown, the problem is to find a self-tuning WMF Kalman filter $\widehat{x}^{(\mathrm{II}) s}(t \mid t)$ for the fused system (2.7) and (2.11). Then, the key to the problem is how to find the consistent estimates of the noise variance and cross-covariance matrices $Q_{w}, S_{i}$, and $R_{i j}$.

\section{Online Estimators of Variances and Cross-Covariances}

Lemma 3.1 (matrix inverse Fadeeva formula [13]). The matrix inverse formula is given by

$$
\left(I_{n}-q^{-1} \Phi\right)^{-1}=\frac{\operatorname{adj}\left(I_{n}-q^{-1} \Phi\right)}{\operatorname{det}\left(I_{n}-q^{-1} \Phi\right)}=\frac{\bar{F}\left(q^{-1}\right)}{\bar{A}\left(q^{-1}\right)}
$$


where $\Phi$ is an $n \times n$ matrix, $q^{-1}$ is a backward shift operator, $I_{n}$ is an $n \times n$ unit matrix, and

$$
\bar{A}\left(q^{-1}\right)=1+\bar{a}_{1} q^{-1}+\cdots+\bar{a}_{n} q^{-n}, \quad \bar{F}\left(q^{-1}\right)=I_{n}+\bar{F}_{1} q^{-1}+\cdots+\bar{F}_{n-1} q^{-(n-1)}
$$

then the coefficients $\bar{a}_{i}$ and $\bar{F}_{i}$ can be computed recursively as

$$
\begin{gathered}
\bar{a}_{i}=-\frac{1}{i} \operatorname{trace}\left(\Phi \bar{F}_{i-1}\right), \quad i=1, \ldots, n, \\
\bar{F}_{i}=\Phi \bar{F}_{i-1}+\bar{a}_{i} I_{n}, \quad i=1, \ldots, n-1, \quad F_{0}=I_{n}, \quad a_{0}=1 .
\end{gathered}
$$

Suppose the greatest common factor of $\bar{A}\left(q^{-1}\right)$ and $\bar{F}\left(q^{-1}\right)$ as scalar polynomial of $\mu\left(q^{-1}\right)$, that is,

$$
\begin{aligned}
& \bar{F}\left(q^{-1}\right)=\mu\left(q^{-1}\right) F\left(q^{-1}\right), \\
& \bar{A}\left(q^{-1}\right)=\mu\left(q^{-1}\right) A\left(q^{-1}\right) .
\end{aligned}
$$

Eliminate the greatest common factor $\mu\left(q^{-1}\right)$ of numerator and denominator in (3.1), we have the irreducible Fadeeva formula:

$$
\begin{gathered}
\left(I_{n}-q^{-1} \Phi\right)^{-1}=\frac{F\left(q^{-1}\right)}{A\left(q^{-1}\right)}, \\
F\left(q^{-1}\right)=I_{n}+F_{1} q^{-1}+\cdots+F_{n_{f}} q^{-n_{f}}, \\
A\left(q^{-1}\right)=1+a_{1} q^{-1}+\cdots+a_{n_{a}} q^{-n_{a}}, \quad a_{n_{a}} \neq 0, n_{a} \leq n-n_{\mu}, \\
F_{i}=\Phi F_{i-1}+a_{i} I_{n}, \quad i=1, \ldots, n_{f}, F_{0}=I_{n}, n_{f}=n-n_{\mu}-1 .
\end{gathered}
$$

Theorem 3.2. For the ith subsystem of systems (2.1) and (2.2) under the Assumptions of 2.1, 2.2, and 2.3 , the innovation model of CARMA

$$
A_{i}\left(q^{-1}\right) y_{i}(t)=C_{i}\left(q^{-1}\right) u(t)+D_{i}\left(q^{-1}\right) \varepsilon_{i}(t)
$$

is stable. The innovation $\varepsilon_{i}(t) \in R^{m_{i}}$ is a white noise with zero mean and variance matrix $Q_{\varepsilon_{i}}$,

$$
\begin{aligned}
& C_{i}\left(q^{-1}\right)=H_{i} F\left(q^{-1}\right) B q^{-1}, \\
& B_{i}\left(q^{-1}\right)=H_{i} F\left(q^{-1}\right) \Gamma q^{-1},
\end{aligned}
$$

where the polynomial matrices of $D_{i}\left(q^{-1}\right), A_{i}\left(q^{-1}\right), C_{i}\left(q^{-1}\right)$, and $B_{i}\left(q^{-1}\right)$ have the form as

$$
X_{i}\left(q^{-1}\right)=X_{i 0}+X_{i 1} q^{-1}+\cdots+X_{i n_{x i}} q^{-n_{x i}}, \quad i=1,2, \ldots, L
$$


with

$$
A_{i 0}=I_{m_{i}}, \quad D_{i 0}=I_{m_{i}}, \quad B_{i 0}=0,
$$

we have

$$
D_{i}\left(q^{-1}\right) \varepsilon_{i}(t)=B_{i}\left(q^{-1}\right) w(t)+A_{i}\left(q^{-1}\right) v_{i}(t)
$$

Proof. From (2.1) and (2.2), we have

$$
y_{i}(t)=H_{i}\left(I_{n}-q^{-1} \Phi\right)^{-1} q^{-1}[B u(t)+\Gamma w(t)]+v_{i}(t)
$$

Applying the extended Fadeeva formula of (3.5), we have

$$
A_{i}\left(q^{-1}\right) y_{i}(t)=C_{i}\left(q^{-1}\right) u(t)+B_{i}\left(q^{-1}\right) w(t)+A_{i}\left(q^{-1}\right) v_{i}(t)
$$

Suppose $\left(A_{i}\left(q^{-1}\right) I_{m_{i}}, C_{i}\left(q^{-1}\right), B_{i}\left(q^{-1}\right)\right)$ left-coprime, and $\left(B_{i}\left(q^{-1}\right), A_{i}\left(q^{-1}\right) I_{m_{i}}\right)$ or their greatest left factor's determinant has no zero point on the unit circles. Note that it needs to be leftcoprime factorization if it is not left-coprime. Then, there is an MA process of $D_{i}\left(q^{-1}\right) \varepsilon_{i}(t)$, which makes (3.10) hold and guarantees $D_{i}\left(q^{-1}\right)$ stable. We have (3.6) from (3.10) and (3.12). The proof is completed.

Define a new measurement process:

$$
z_{i}(t)=A_{i}\left(q^{-1}\right) y_{i}(t)-C_{i}\left(q^{-1}\right) u(t)
$$

From (3.6), we have

$$
z_{i}(t)=D_{i}\left(q^{-1}\right) \varepsilon_{i}(t)
$$

From (3.10), we have

$$
z_{i}(t)=B_{i}\left(q^{-1}\right) w(t)+A_{i}\left(q^{-1}\right) v_{i}(t)
$$

Remark 3.3. When the noise variances $Q_{w}$ and $R_{i}$ are known, the Gevers-Wouters [20] algorithm can be used to construct ARMA innovation model and obtain $D_{i}\left(q^{-1}\right)$ and $Q_{\varepsilon_{i}}$. 
In (3.14), $z_{i}(t)$ is a stationary stochastic process, whose correlated function $R_{z_{i j}}(k)$ has cut-off property, and suppose it be cut-off at $n_{z_{i j}}$, that is,

$$
\begin{gathered}
R_{z_{i j}}(k)=\mathrm{E}\left[z_{i}(t) z_{j}^{\mathrm{T}}(t-k)\right], \quad k=0,1, \ldots, n_{z_{i j}} \\
R_{z_{i j}}(k)=0, \quad k>n_{z_{i j}}, \quad i, j=1,2, \ldots, L .
\end{gathered}
$$

At the end of time $t$, the sampling estimation of the correlated function $R_{z_{i j}}(k)$ ( $k=$ $\left.0,1, \ldots, n_{z_{i j}}\right)$ based on measurements $\left(z_{i}(t), z_{i}(t-1), z_{i}(t-2), \ldots\right)$ can be defined as

$$
\widehat{R}_{z_{i j}}^{t}(k)=\frac{1}{t} \sum_{\alpha=1}^{t} z_{i}(\alpha) z_{j}^{\mathrm{T}}(\alpha-k)
$$

then we have its recursive form:

$$
\widehat{R}_{z_{i j}}^{t}(k)=\widehat{R}_{z_{i j}}^{t-1}(k)+\frac{1}{t}\left[z_{i}(t) z_{j}^{\mathrm{T}}(t-k)-\widehat{R}_{z_{i j}}^{t-1}(k)\right], \quad t=2,3, \ldots,
$$

with the initial value $\widehat{R}_{z_{i j}}^{1}(k)=z_{i}(1) z_{j}^{\mathrm{T}}(1-k)$.

Computing the correlated function on both sides of (3.14), we have

$$
\begin{array}{r}
R_{z_{i j}}(k)=\sum_{u=k}^{n_{0}} B_{i u} Q_{w} B_{j, u-k}^{\mathrm{T}}+\sum_{u=k}^{n_{0}} A_{i u} S_{i}^{\mathrm{T}} B_{j, u-k}^{\mathrm{T}}+\sum_{u=k}^{n_{0}} B_{i u} S_{j} A_{j, u-k}^{\mathrm{T}}+\sum_{u=k}^{n_{0}} A_{i u} R_{i j} A_{j, u-k}^{\mathrm{T}}, \\
k=0,1, \ldots, n_{z_{i j}}, \quad i, j=1, \ldots, L,
\end{array}
$$

where $n_{0}=\max \left(n_{a i}, n_{b i}, n_{a j}, n_{b j}\right) . A_{i j}$ and $B_{i j}$ are known, $B_{i j}=0\left(j>n_{b i}\right), A_{i j}=0\left(j>n_{a i}\right)$.

However (3.19) is a matrix equations set. Substituting the sampling estimates at the time $t \widehat{R}_{z_{i j}}^{t}(k)\left(k=0, \ldots, n_{z_{i j}}\right)$ into (3.19), and solving the matrix equations set, then we have the estimates $\widehat{S}_{i}(t), \widehat{Q}_{w}(t)$, and $\widehat{R}_{i j}(t)$ at the time $t$.

From the ergodicity of the stationary stochastic process (3.14) and the Assumption 2.3, when $t \rightarrow \infty$, we have $\widehat{S}_{i}(t) \rightarrow S_{i}, \widehat{Q}_{w}(t) \rightarrow Q_{w}$ and $\widehat{R}_{i j}(t) \rightarrow R_{i j}$.

\section{Self-Tuning WMF Kalman Filter}

When the statistical features of the noise are unknown, the self-tuning weighted measurement fusion Kalman estimator can be obtained through the following three steps.

Step 1. For different sensor systems, (3.16)- (3.19) are used to identify online the estimates $\widehat{Q}_{w}(t), \widehat{R}_{i j}(t)$, and $\widehat{S}_{i}(t), i, j=1, \ldots, L$, of noise variances of $Q_{w}, R_{i j}$, and $S_{i}$ at the time $t$, which 
will yield the following available estimates at the time $t$ :

$$
\begin{aligned}
& \widehat{S}=\left[\widehat{S}_{1}, \ldots, \widehat{S}_{L}\right], \\
& \widehat{R}^{(\mathrm{I})}=\left[\begin{array}{ccc}
\widehat{R}_{11} & \cdots & \widehat{R}_{1 L} \\
\vdots & & \vdots \\
\widehat{R}_{L 1} & \cdots & \widehat{R}_{L L}
\end{array}\right], \\
& \widehat{J}=\Gamma \widehat{S} \widehat{R}^{(\mathrm{I})-1}, \\
& \widehat{\bar{u}}(t)=B u(t)+\widehat{J} y^{(\mathrm{I})}(t), \\
& \widehat{Q}=\widehat{Q}_{w}-\widehat{S} \widehat{R}^{(\mathrm{I})-1} \widehat{S}^{\mathrm{T}}, \quad \hat{\bar{Q}}_{w}=\Gamma \widehat{Q} \Gamma^{\mathrm{T}}, \\
& \widehat{y}^{(I I)}(t)=\widehat{\Omega} y^{(I)}(t), \\
& \widehat{\Omega}=\left(F^{\mathrm{T}} \widehat{R}^{(\mathrm{I})-1} F\right)^{-1} F^{\mathrm{T}} \widehat{R}^{(\mathrm{I})-1}, \\
& \widehat{R}^{(\mathrm{II})}=\left(F^{\mathrm{T}} \widehat{R}^{(\mathrm{I})-1} F\right)^{-1}, \\
& \widehat{\bar{\Phi}}=\Phi-\widehat{J} H^{(\mathrm{I})} \text {. }
\end{aligned}
$$

Step 2. From (2.15), solving the following Riccati equation, we get the estimation value $\widehat{\Sigma}^{\text {(II) }}$ of $\Sigma^{(\mathrm{II})}$ at the time $t$ :

$$
\widehat{\Sigma}^{(\mathrm{II})}=\hat{\bar{\Phi}}\left[\widehat{\Sigma}^{(\mathrm{II})}-\widehat{\Sigma}^{(\mathrm{II})} H^{(\mathrm{II}) \mathrm{T}}\left(H^{(\mathrm{II})} \widehat{\Sigma}^{(\mathrm{II})} H^{(\mathrm{II}) \mathrm{T}}+\widehat{R}^{(\mathrm{II})}\right)^{-1} H^{(\mathrm{II})} \widehat{\Sigma}^{(\mathrm{II})}\right] \widehat{\bar{\Phi}}^{\mathrm{T}}+\hat{\bar{Q}}_{w} .
$$

Step 3. Equations (2.14) are applied, and the self-tuning weighted measurement fusion state estimator is given by

$$
\begin{aligned}
& \widehat{x}^{(\mathrm{II}) s}(t+1 \mid t+1)=\widehat{x}^{(\mathrm{II}) s}(t+1 \mid t)+\widehat{K}_{f}^{(\mathrm{II})} \widehat{\varepsilon}^{(\mathrm{II})}(t+1), \\
& \widehat{x}^{(\mathrm{II}) s}(t+1 \mid t)=\widehat{\Psi}_{p}^{(\mathrm{II})} \widehat{x}^{(\mathrm{II}) s}(t \mid t-1)+\widehat{\bar{K}}_{p}^{(\mathrm{II})} \widehat{y}^{(\mathrm{II})}(t)+\hat{\bar{u}}(t), \\
& \widehat{x}^{(\mathrm{II}) s}(t \mid t+N)=\widehat{x}^{(\mathrm{II}) s}(t \mid t)+\sum_{j=1}^{N} \widehat{K}^{(\mathrm{II})}(j) \widehat{\varepsilon}^{(\mathrm{II})}(t+j), \quad N>0, \\
& \widehat{\varepsilon}^{(\mathrm{II})}(t)=\widehat{y}^{(\mathrm{II})}(t)-H^{(\mathrm{II})} \widehat{x}^{(\mathrm{II}) s}(t \mid t-1), \\
& \widehat{K}_{f}^{(\mathrm{II})}=\widehat{\Sigma}^{(\mathrm{II})} H^{(\mathrm{II}) \mathrm{T}}\left(H^{(\mathrm{II})} \widehat{\Sigma}^{(\mathrm{II})} H^{(\mathrm{II}) \mathrm{T}}+\widehat{R}^{(\mathrm{II})}\right)^{-1} \text {, } \\
& \widehat{\bar{K}}_{p}^{(\mathrm{II})}=\widehat{\bar{\Phi}} \widehat{\Sigma}^{(\mathrm{II})} H^{(\mathrm{II}) \mathrm{T}}\left(H^{(\mathrm{II})} \widehat{\Sigma}^{(\mathrm{II})} H^{(\mathrm{II}) \mathrm{T}}+\widehat{R}^{(\mathrm{II})}\right)^{-1}, \\
& \widehat{\Psi}_{p}^{(\mathrm{II})}=\widehat{\bar{\Phi}}-\widehat{\bar{K}}_{p}^{(\mathrm{II})} H^{(\mathrm{II})}, \\
& \widehat{K}^{(\mathrm{II})}(j)=\widehat{\Sigma}^{\mathrm{II}}\left(\left(I_{n}-\widehat{K}_{f}^{(\mathrm{II})} H^{(\mathrm{II})}\right)^{\mathrm{T}} \widehat{\bar{\Phi}}^{\mathrm{T}}\right)^{j} H^{(\mathrm{II}) \mathrm{T}}\left(H^{(\mathrm{II})} \widehat{\Sigma}^{(\mathrm{II})} H^{(\mathrm{II}) \mathrm{T}}+\widehat{R}^{(\mathrm{II})}\right)^{-1} \text {, } \\
& \widehat{P}^{(\mathrm{II})}=\left[I_{n}-\widehat{K}_{f}^{(\mathrm{II})} H^{(\mathrm{II})}\right] \widehat{\Sigma}^{(\mathrm{II})} .
\end{aligned}
$$




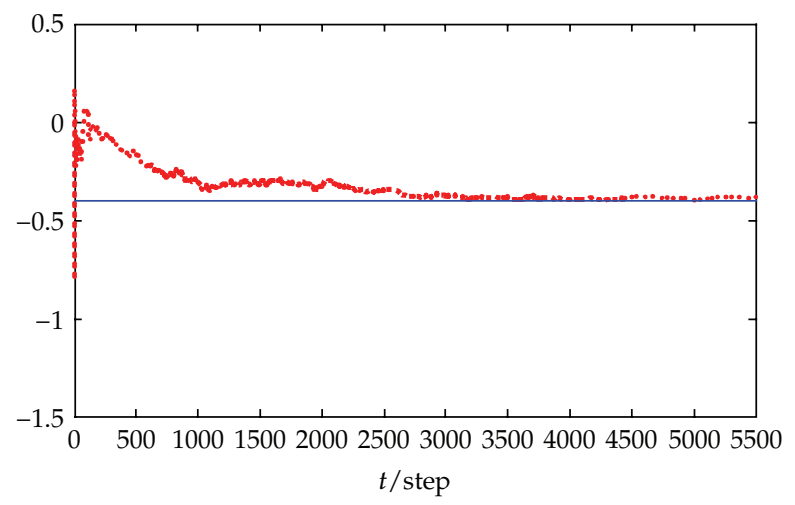

Figure 1: $\rho_{1}$ and the convergence of estimation value $\widehat{\rho}_{1}(t)$.

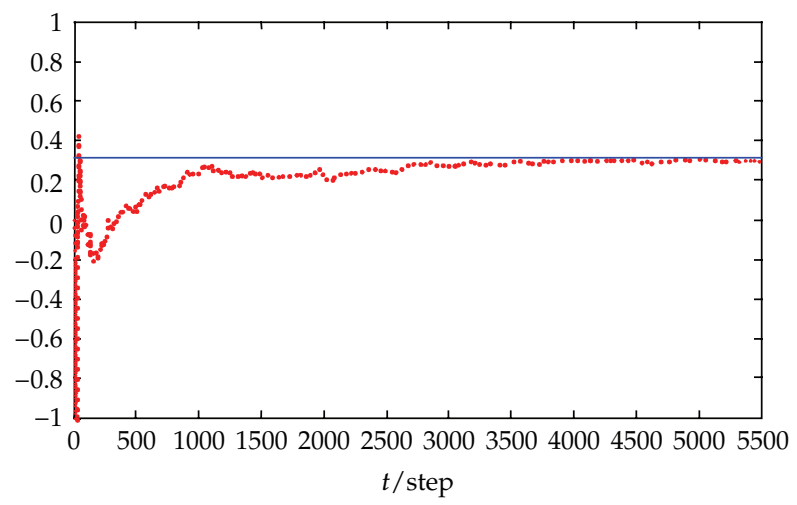

Figure 2: $d_{11}$ and the convergence of estimation value $\widehat{d}_{11}(t)$.

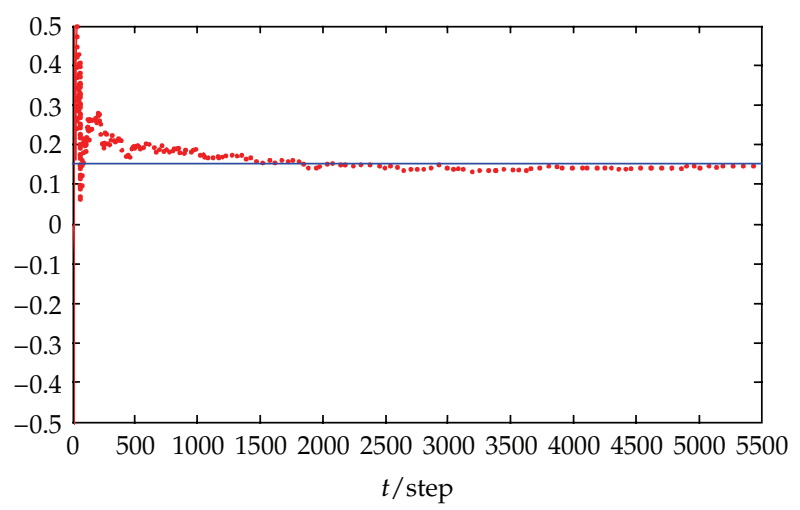

Figure 3: $d_{12}$ and the convergence of estimation value $\widehat{d}_{12}(t)$.

Remark 4.1. At every moment, the iteration method to solve Riccati equation (4.2) causes comparatively large computation burden, which is not convenient for the real applications. To reduce the computational burden, a computing period (dead band) $T_{d}$ of (4.2) is set and the estimation value $\widehat{\Sigma}^{(I I)}$ keeps invariant in the period $T_{d}$. So, $\widehat{\Sigma}^{(I I)}$ is only computed at the moments of $t=T_{d}, 2 T_{d}, 3 T_{d}, \ldots$, which can reduce computation burden, and can be called Riccati equation with a dead band $[9,17]$. 


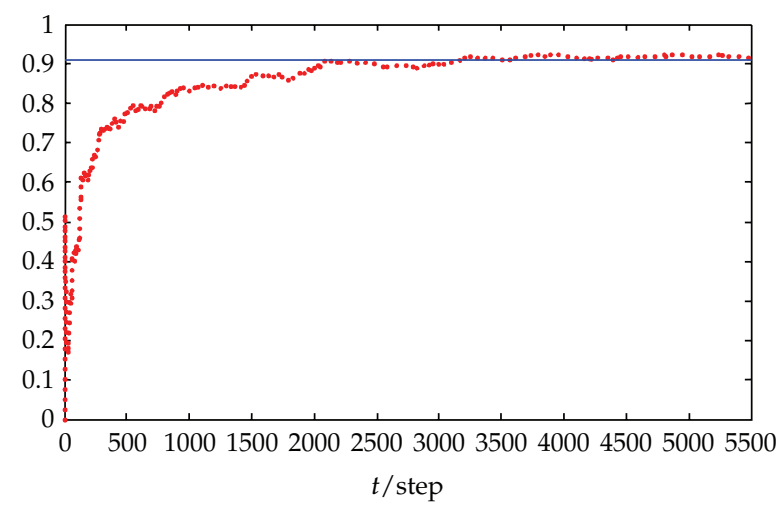

Figure 4: $Q_{\varepsilon_{1}}$ and the convergence of estimation value $\widehat{Q}_{\varepsilon_{1}}(t)$.

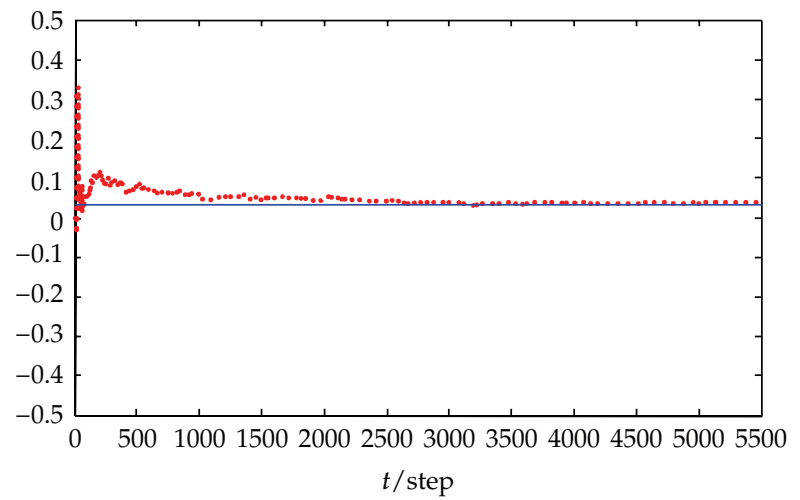

Figure 5: $Q_{\xi_{1}}$ and the convergence of estimation value $\widehat{Q}_{\xi_{1}}(t)$.

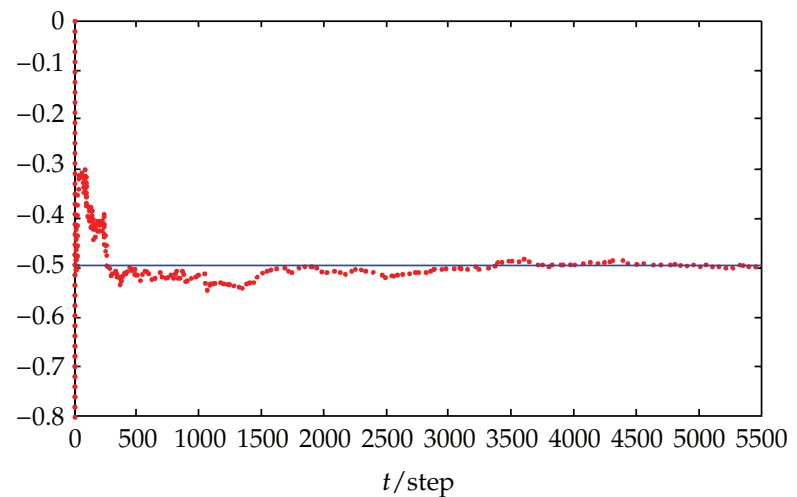

Figure 6: $\rho_{2}$ and the convergence of estimation value $\widehat{\rho}_{2}(t)$. 


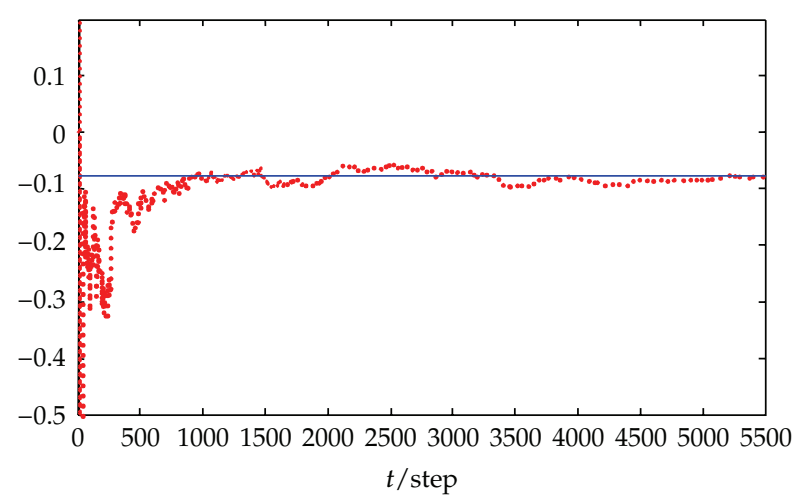

Figure 7: $d_{21}$ and the convergence of estimation value $\widehat{d}_{21}(t)$.

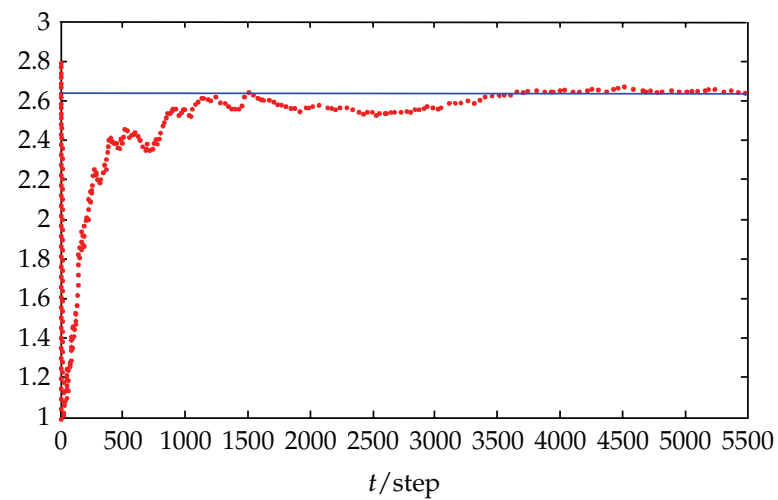

Figure 8: $Q_{\varepsilon_{2}}$ and the convergence of estimation value $\widehat{Q}_{\varepsilon_{2}}(t)$.

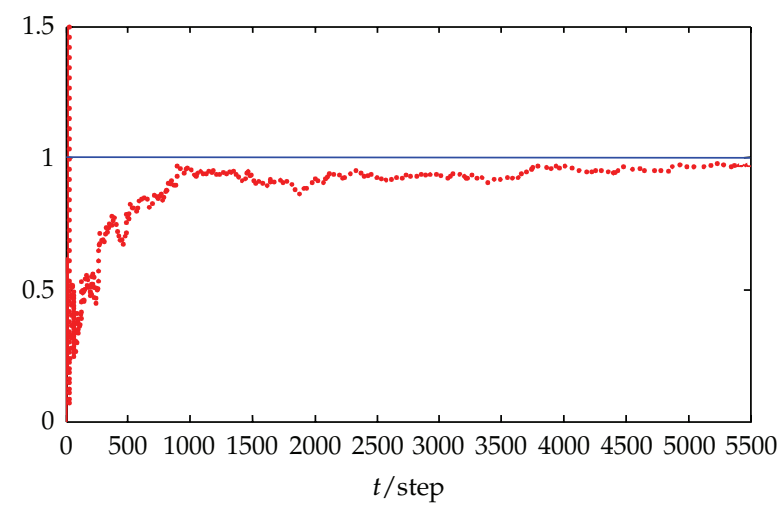

Figure 9: $Q_{w}$ and the convergence of estimation value $\widehat{Q}_{w}(t)$. 


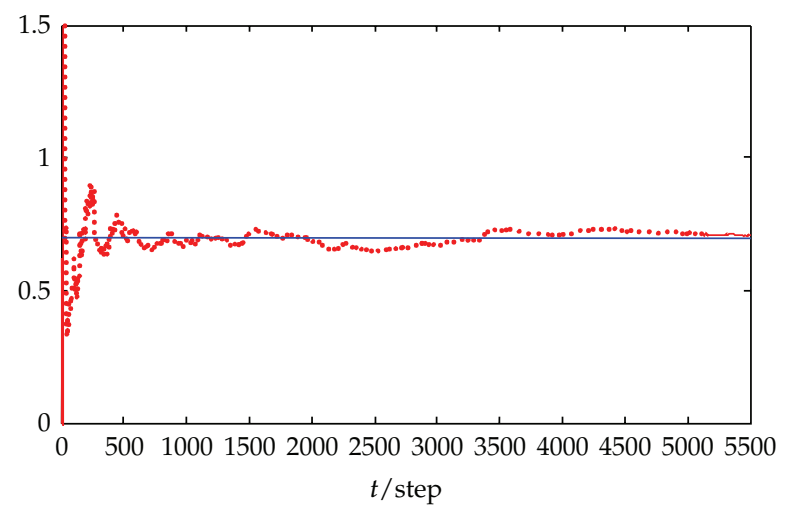

Figure 10: $Q_{\xi_{2}}$ and the convergence of estimation value $\widehat{Q}_{\xi_{2}}(t)$.

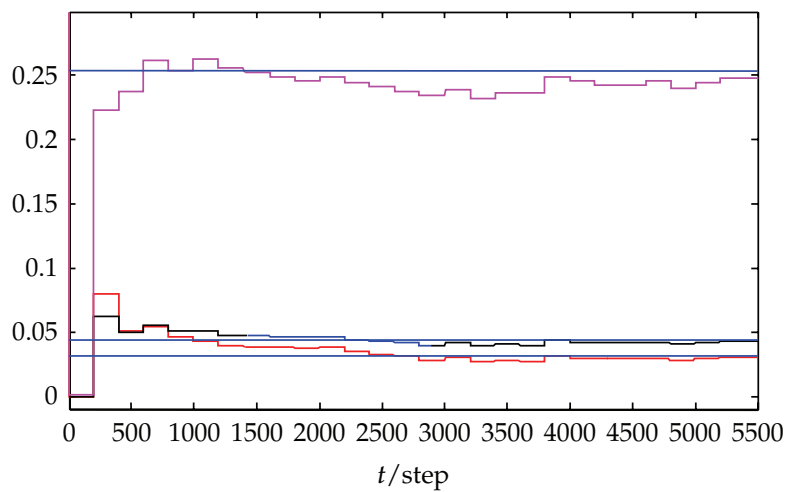

Figure 11: $\Sigma^{(\mathrm{II})}$ and the convergence of estimation value $\widehat{\Sigma}^{(\mathrm{II})}(t)$.

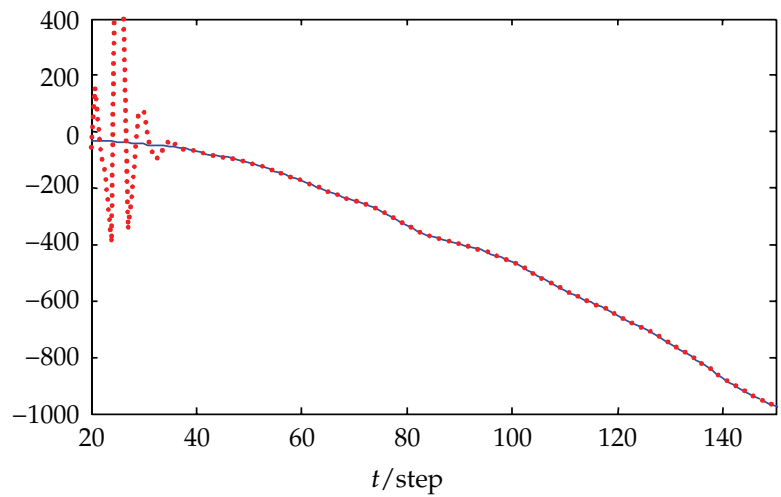

Figure 12: Positon $x_{1}$ and self-tuning WMF Kalman filter $\widehat{x}_{1}^{(\mathrm{II}) s}(t \mid t)$. 


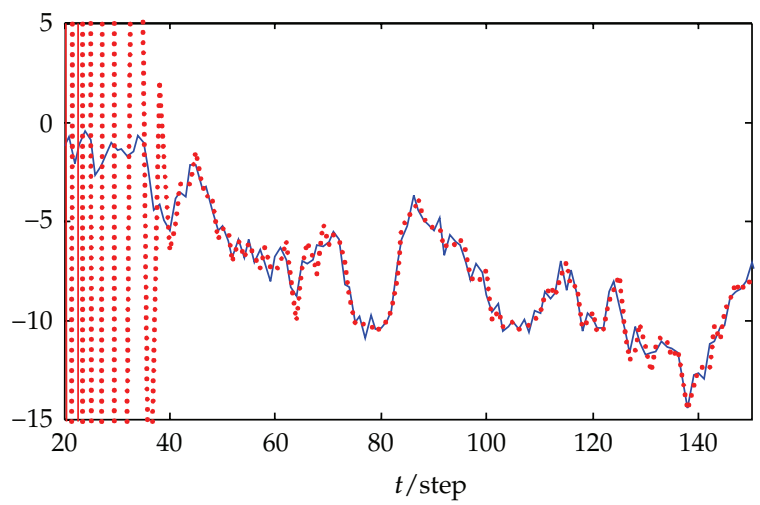

Figure 13: Speed $x_{2}$ and self-tuning WMF Kalman filter $\widehat{x}_{2}^{(\mathrm{II}) s}(t \mid t)$.

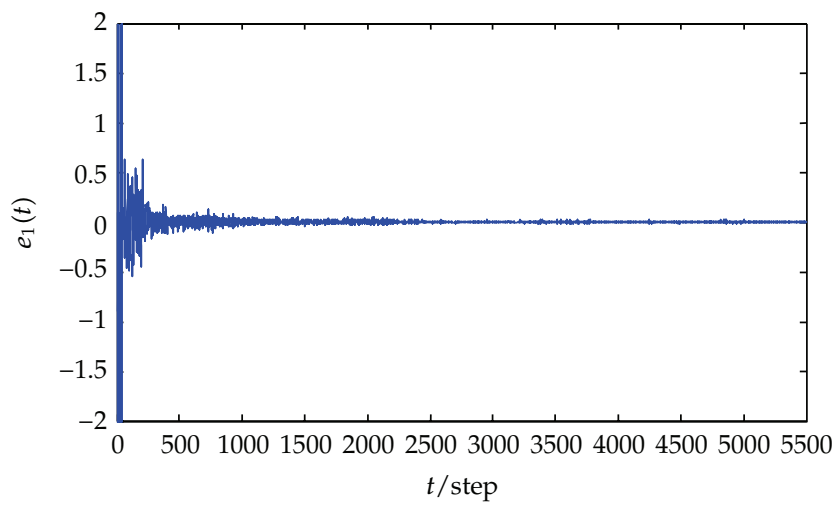

Figure 14: The curve of position errors $e_{1}(t)=\widehat{x}_{1}^{(\mathrm{II})}(t \mid t)-\widehat{x}_{1}^{(\mathrm{II}) s}(t \mid t)$ for optimal and self-tuning WMF Kalman filter.

\section{Simulation Example}

The self-tuning $\alpha-\beta$ radar track system with colored measurement noise:

$$
\begin{gathered}
x(t+1)=\left[\begin{array}{cc}
1 & T_{0} \\
0 & 1
\end{array}\right] x(t)+\left[\begin{array}{c}
0.5 T_{0}^{2} \\
T_{0}
\end{array}\right] w(t), \\
y_{i}(t)=H_{0 i} x(t)+v_{i}(t), \quad i=1,2 \\
v_{i}(t+1)=\rho_{i} v(t)+\xi_{i}(t)
\end{gathered}
$$

where $w(t)$ and $\xi_{i}(t), i=1,2$, are independent Gaussian white noises with zero means and variances of $Q_{w}=1, Q_{\xi_{1}}=0.04$, and $Q_{\xi_{2}}=0.7, v_{i}(t)$ is colored measurement noise, $H_{01}=$ $\left[\begin{array}{ll}1 & 0\end{array}\right]$ and $H_{02}=\left[\begin{array}{ll}0 & 1\end{array}\right] . T_{0}=1$ is the sampling period, $x(t)=\left[x_{1}(t) x_{2}(t)\right]^{\mathrm{T}}, x_{1}(t)$ and $x_{2}(t)$, are, respectively, the position and speed of the moving object at $t T_{0}, \rho_{1}=-0.4, \rho_{2}=-0.5$.

When $\rho_{i}, Q_{w}$ and $Q_{\xi_{i}}(i=1,2)$ are unknown, the problem is to find the self-tuning WMF $\alpha-\beta$ tracking filter $\widehat{x}^{(\mathrm{II}) s}(t \mid t)$.

The parameter convergence results of subsystem 1 are shown in Figures 1, 2, 3, 4, and 5 , and the parameter convergence results of subsystem 2 are shown in Figures 6, 7, 8, 9, and 10, 


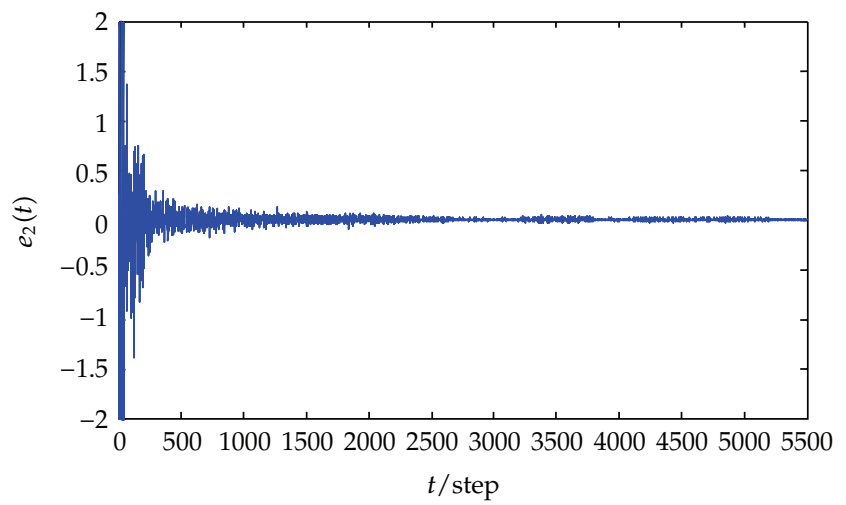

Figure 15: The curve of velocity errors $e_{2}(t)=\widehat{x}_{2}^{(\mathrm{II})}(t \mid t)-\widehat{x}_{2}^{(\mathrm{II}) s}(t \mid t)$ for optimal and self-tuning WMF Kalman filter.

where the curved lines denote the estimates and the straight lines denote true values. The self-tuning WMF Kalman filter $\widehat{x}^{(\mathrm{II}) s}(t \mid t)$ is shown in Figures 11, 12, 13, 14, and 15, where Figure 11 is the convergence of $\widehat{\Sigma}^{(I I)}$. Using the iteration algorithm with dead band $T_{d}=200$, we can see that the parameter estimates in $\widehat{\Sigma}^{\text {(II) }}$ shown in ladder-shape curves converge to the corresponding real values.

The object-tracking curves of self-tuning WMF Kalman filter is shown in Figures 12, 13, 14 , and 15 . We can see that the system can gradually track the position and speed of the objects as the time increases, where the real lines are real values and the dashed lines are estimates. The error curves between the optimal and self-tuning WMF Kalman filter is shown in Figures 14 and 15 . We see that the error curves take on the funnel shape, which demonstrates that the self-tuning filter has better convergence.

\section{Conclusion}

For multisensor linear discrete time-invariant stochastic control system with different measurement matrices and correlated noise, an online identification method is designed when the input noise and measurement noise variance are unknown. It firstly uses Fadeeva formula to construct ARMA innovation model with unknown noise covariance matrices, and then uses the ergodicity of sampling-correlated function in the stationary and inverse ARMA innovation model to identify the noise covariance matrices. Further, a self-tuning WMF Kalman filter has been presented from a steady-state global optimal measurement fusion Kalman filter by matrix full-rank decompostion, weighted least squares method, and measurement feedback. It has asymptotic global optimality. Compared to the centralized fusion algorithm, it can reduce the computational burden.

\section{Acknowledgments}

This work is supported by National Natural Science Foundation of China under Grant NSFC 61174139, Key Project of Chinese Ministry of Education under Grant 209038, Key Laboratory of Electronics Engineering, College of Heilongjiang Province under Grant DZZD 20100038, 
Science and Technology Research Foundation of Heilongjiang Province Education Department under Grant 12521420, Open Laboratory Project of Heilongjiang University in China 2012, and High Level Talents Support Project of Heilongjiang University in China under Grant Hdtd2010-03.

\section{References}

[1] Y. B. Shalom, "On the track-to-track correlation problem," IEEE Transactions on Automatic Control, vol. 26, no. 2, pp. 571-572, 1981.

[2] Y. B. Shalom and X.-R. Li, Multigarget-Multisensor Tracking: Principles and Techniques, Yaakov BarShalom Publishing, Storrs, Conn, USA, 1995.

[3] N. A. Carlson, "Federated square root filter for decentralized parallel processes," IEEE Transactions on Aerospace and Electronic Systems, vol. 26, pp. 517-525, 1990.

[4] K. H. Kim, "Development of track to track fusion algotithm," in Proceeding of the American Control Conference, pp. 1037-1041, Baltimore, Md, USA, 1994.

[5] X. R. Li, Y. Zhu, J. Wang, and C. Han, "Optimal linear estimation fusion-part I: unified fusion rules," IEEE Transactions on Information Theory, vol. 49, no. 9, pp. 2192-2323, 2003.

[6] S.-L. Sun and Z.-L. Deng, "Multi-sensor optimal information fusion Kalman filter," Automatica, vol. 40, no. 6, pp. 1017-1023, 2004.

[7] S.-L. Sun, "Multi-sensor information fusion white noise filter weighted by scalars based on Kalman predictor," Automatica, vol. 40, no. 8, pp. 1447-1453, 2004.

[8] Q. Gan and J. H. Chris, "Comparison of two measurement fusion methods for Kalman filter based multisensor data fusion," IEEE Transactions on Aerospace and Electronic Systems, vol. 37, no. 1, pp. 273279, 2001.

[9] Y. Gao, W. J. Jia, X. J. Sun, and Z. L. Deng, "Self-tuning multisensor weighted measurement fusion kalman filter," IEEE Transactions on Aerospace and Electronic Systems, vol. 45, no. 1, pp. 179-191, 2009.

[10] X. Wang, Q. D. Zhu, and Y. B. Wu, "A measurement fusion fault-tolerating PID control for time-delay system with colored noise disturbance," Key Engineering Materials, vol. 419-420, pp. 589-592, 2010.

[11] X. Wang, Q. D. Zhu, and S. L. Sun, "Weighted measurement fusion estimation algorithm with correlated noises and its global optimality," Systems Engineering and Electronics, vol. 32, no. 10, pp. 20572061, 2010.

[12] X. Wang, Q.-D. Zhu, and S.-L. Sun, “Universal weighted measurement fusion estimation algorithm and its global optimality," Computer Engineering and Applications, vol. 46, no. 24, pp. 22-25, 2010.

[13] Z.-L. Deng, Self-Tuning Filtering Theory with Application, Harbin institute of Technology Press, Harbin, China, 2003.

[14] S. Sun, "Optimal and self-tuning information fusion Kalman multi-step predictor," IEEE Transactions on Aerospace and Electronic Systems, vol. 43, no. 2, pp. 418-427, 2007.

[15] Z.-L. Deng, Y. Gao, C.-B. Li, and G. Hao, "Self-tuning decoupled information fusion Wiener state component filters and their convergence," Automatica, vol. 44, no. 3, pp. 685-695, 2008.

[16] Y. Gao, C.-J. Ran, and Z.-L. Deng, "Weighted measurement fusion Kalman filter with correlated measurement noises and its global optimality," in Proceedings of the International Colloquium on Information Fusion, pp. 228-234, 2007.

[17] C. J. Ran and L. Z. Deng, "Self-tuning weighted measurement fusion Kalman filtering algorithm," Computational Statistics and Data Analysis, vol. 56, pp. 2112-2128, 2012.

[18] R. A. Horn and C. R. Johnson, Matrix Analysis, Cambridge University Press, Cambridge, 1985.

[19] T. A. Kailath, H. Sayed, and B. Hassibi, Linear Estimation, Prentice-Hall, Upper Saddle River, NJ, USA, 2000.

[20] Z.-L. Deng and X. Wang, Modeling and Estimation, Science Press, Beijing, China, 2007.

[21] Z.-L. Deng, Information Fusion Filtering Theory with Applications, Harbin institute of Technology Press, Harbin, China, 2007. 


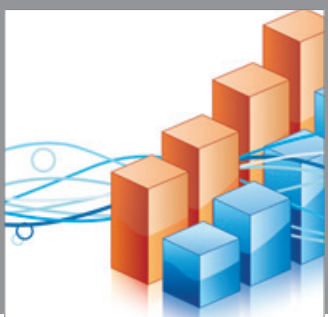

Advances in

Operations Research

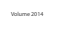

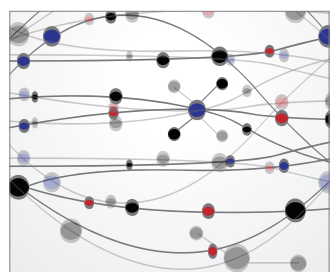

\section{The Scientific} World Journal
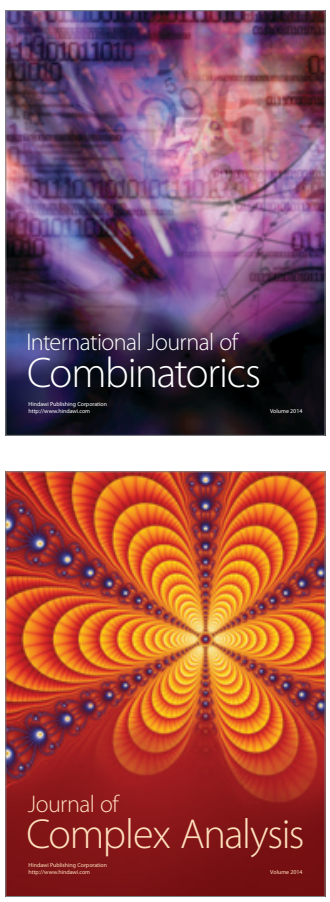

International Journal of

Mathematics and

Mathematical

Sciences
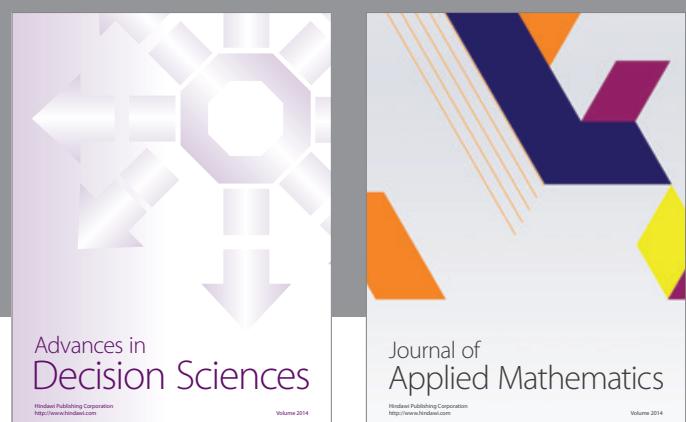

Journal of

Applied Mathematics
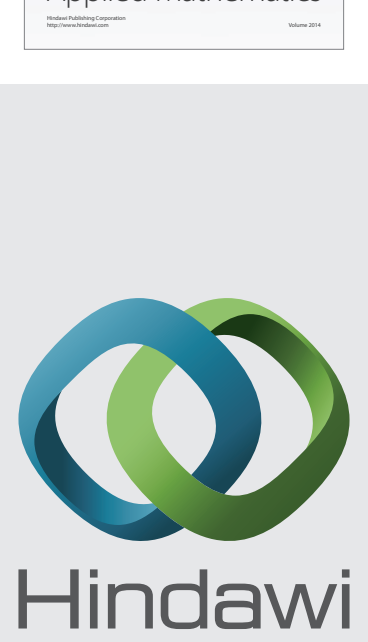

Submit your manuscripts at http://www.hindawi.com
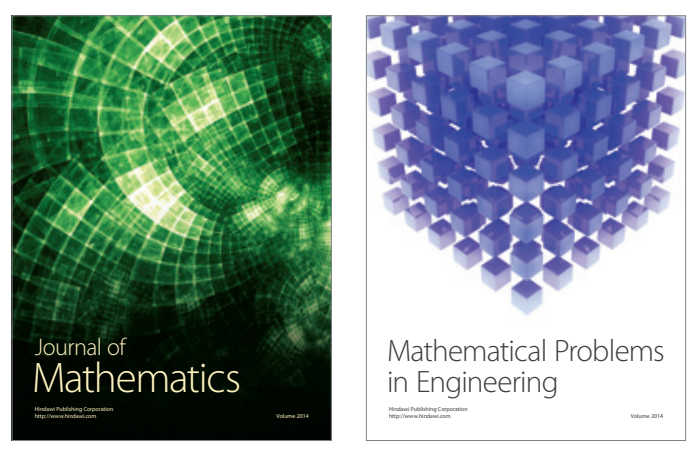

Mathematical Problems in Engineering
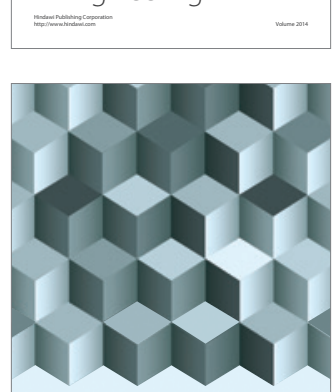

Journal of

Function Spaces
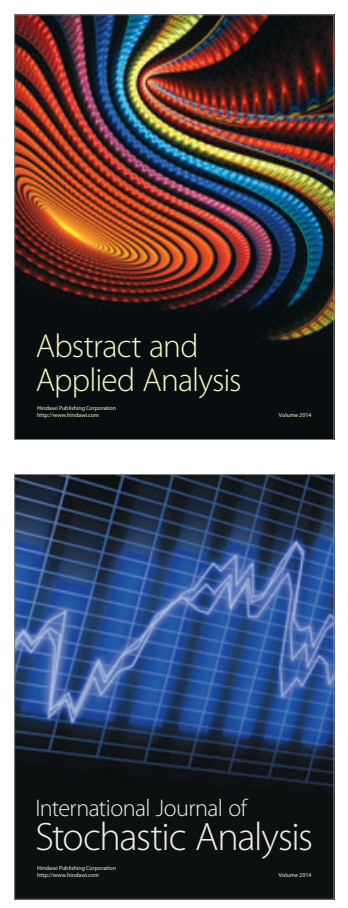

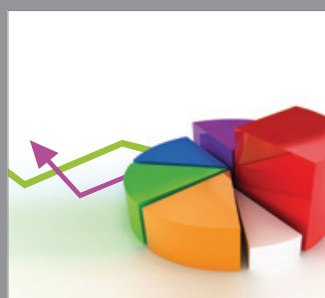

ournal of

Probability and Statistics

Promensencen
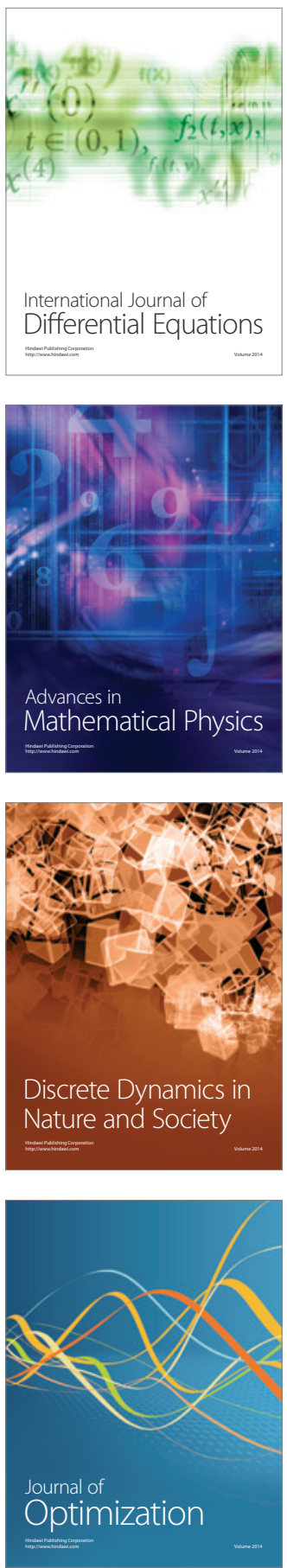